\title{
Fiber optic communications experiments for undergraduates
}

\section{Brian Culshaw, Walter Johnstone, George Stewart, Douglas Walsh}

Brian Culshaw, Walter Johnstone, George Stewart, Douglas Walsh, "Fiber optic communications experiments for undergraduates," Proc. SPIE 2525, 1995 International Conference on Education in Optics, (13 October 1995); doi: $10.1117 / 12.224050$

Event: SPIE's 1995 International Symposium on Optical Science, Engineering, and Instrumentation, 1995, San Diego, CA, United States 
Fibre optic communications experiments for undergraduates

\author{
Brian Culshaw, Walter Johnstone, George Stewart and *Douglas Walsh \\ University of Strathclyde, Department of Electronic \& Electrical Engineering, 204 George Street \\ Glasgow G1 1XW \\ *OptoSci Ltd, Strathclyde University Incubator, 141 St James Road, Glasgow G4 OLT
}

\begin{abstract}
$\underline{\text { ABSTRACT }}$
The majority of undergraduate courses in electronic and electrical engineering include some element of optoelectronics particularly fibre optics for communications. The majority of tutors prefer to include experimental demonstrations of underlying concepts within the course structure but for fibre optics this is difficult to achieve within a realistic teaching budget. This paper describes our experiences at Strathclyde in providing experimental back-up to such courses through a simple waveguide experiment and an experimental simulation of an optical communication system demonstrating the principal features thereof. The two experiments, which are designed to be incorporated in the third year of an undergraduate four or five year programme, illustrate the concepts of modes, polarisation dependence in thin films, the difference between light emitting diode and laser diode, source performance in optical communications and the influence of material and intermode dispersion on system performance. They also examine noise limitation and the potential bit error rate performances which the system offers. They are designed to fit within a realistic budget and are configured as mini projects and occupy a few days of laboratory time.
\end{abstract}

\title{
1. INTRODUCTION
}

Modern undergraduate degree courses in electronic and electrical engineering all include significant elements of optoelectronics with emphasis upon engineering applications, particularly communications, rather than upon underlying physical principles. There are also similar needs for applications oriented credits in courses in computing and information technology, applied physics and in more general degree courses which offer combinations of technology and business and which are offered many institutions world-wide.

It is simplest to teach these courses on an entirely theoretical basis but from an engineering perspective this is extremely unsatisfactory since the objective must be to be able to convey the application and usefulness of optical concepts in engineering rather than simply the way in which things work. The dilemma which confronts the educationalist is that whilst these objectives are desirable - even essential - their implementation is thwarted by the extremely high cost of the typical equipment which is used by the engineer in the field. This may be excellent for demonstrating a cost benefit analysis and indicating to students how, despite this extremely expensive equipment, the cost per bit.kilometer for information has plummeted by orders of magnitude of orders of magnitude over the past quarter of a century. However, it precludes the use of such equipment in undergraduate teaching laboratories.

At Strathclyde we have approached this dilemma by experimental rather than theoretical simulation of system performance, based upon critical analysis of a greatly simplified communication system 
which enables the demonstration of all the phenomena in optical communications (attenuation, dispersion etc) within a realistic budget. We have complemented this system oriented experiment with a waveguide demonstration within which the students fabricate a very simple ion exchange waveguide and use this to seek out the characteristics of propagating modes and their relationship with waveguide thickness and index differences. These experiments are both configured as "mini projects" and feature within the 3 rd year of a $4 / 5$ year engineering undergraduate curriculum.

\section{ENGINEERING EDUCATION IN SCOTLAND}

Scotland differs somewhat from the remainder of the United Kingdom in its higher education provision and as such a short comment on course structures is appropriate. The normal honours degree curriculum in Scotland extends over a period of 4 years: the BEng Honours from a Scottish University is broadly equivalent to the 3 year BEng Honours from an English University. Scottish students arrive in the university with a school leaving certificate (the Scottish Highers) usually encompassing 5 subjects, one of which is English, in addition to the more traditional science oriented disciplines.

Within the UK the BEng is usually a technically oriented degree with an emphasis upon a specific engineering discipline. There is an increasing trend within UK higher education in engineering towards the MEng qualification which integrates in essence the technical elements of the BEng with extensive supplementary exposure to management and languages (though the BEng degree does include elements of this).

At Strathclyde the course structure is credit based. For BEng students about $15 \%$ of the course is non-technical and within the current European environment, many students opt to take a language option - we are gradually increasing our output of young engineers fluent in a second language. Many of our BEng undergraduates avail themselves of the opportunity to study for one semester at a partner institution within Europe where they are taught in the host language. Strathclyde also offers two MEng programmes. One has a strong business emphasis but with provision for language elements at the level of the BEng and the second emphasises the European connection embracing within its structure a whole year in a partner institution (INSA in Rennes, France) and a dual qualification awarded by both partner institutions on graduation. The MEng course in Scotland extends over 5 years and is largely compatible with many of the engineering diploma courses offered within continental Europe.

\section{OPTICAL COMMUNICATIONS FOR UNDERGRADUATES - THE BASIC PROBLEM}

Optical communications is the principal feature within our third year introductory course on optical electronics. There are three overall contributory concepts covered within the course :

- The properties of components including laser diodes, light emitting diodes, photodetectors, optical fibre amplifiers and optical fibre waveguides.

- System performance criteria including transmission distances and bit error rate concepts 
- System design concepts and system applications including power and dispersion budgets.

These concepts are covered at a theoretical level (with the possible exception of optical fibre amplifiers) in every undergraduate text on optical fibre communications and at Strathclyde these texts are complemented by extensive course notes. Experimental demonstrations are, however, somewhat unusual and we felt that it was essential for students to be able to become familiar with these underlying concepts in a practical context. The course structure within our third year curriculum also includes provision for extended laboratories or mini projects which are programmed to take 4-8 half days of laboratory time and which furnish the ideal framework within which to undertake the guided investigative experiments which are essential to highlight these concepts.

\section{EXPERIMENTAL INTRODUCTION TO OPTICAL WAVEGUIDES}

The principal aim of this experimental demonstration is to introduce the concepts of modes in optical waveguides.

The first stage is to fabricate a simple planar guide using ion exchange and a microscope slide. The next phase is to prism couple light from the helium neon laser into this guide and examine the modal launch conditions both as function of angle between the input laser beam and the microscope slide substrate and input state of polarisation. This stage of the experiment provides insight to both the concepts of resonant coupling and the ideas of intermode dispersion (through geometrical arguments). It also illustrates the polarisation sensitivity of thin dielectric films.

The waveguides are fabricated by placing them in a silver nitrate/sodium nitrate melt comprising $1 \%$ by weight of silver nitrate. This operation is performed in a simple tube furnace operating at about $315^{\circ} \mathrm{C}$. A variety of slides may be inserted into the furnace for different times in order to fabricate waveguides of thickness varying from a few to about $10 \mu \mathrm{m}$. At this stage no direct measurements are made of waveguide thickness as this is inferred from the mode spectrum measurements.

The optical equipment is very simple and is illustrated in Figure 1. Rotating the stage (for both TE and TM launch polarisations) enables measurements of both the effective index of individual modes and the number of modes propagated in the film for the helium neon wavelength of $663 \mathrm{~nm}$. The effective index for a particular mode is obtained from :

$$
n_{e}=n_{p} \sin \left[A+\sin ^{-1}\left(\frac{\sin \theta_{m}}{n_{p}}\right)\right]
$$

where $n_{e}$ is the effective index of the guided mode, $n_{p}$ is the index of the prism (1.6893), $\theta_{m}$ is the launch angle (see Figure 2) and $\mathrm{A}$ is the angle between the waveguide surface and the input phase of the prism (in our case $60^{\circ}$.

In order to estimate the diffusion depth of the guide a linear profile of depth d (figure 3 ) is assumed together with a small index difference and the well known dispersion relationships are used : 


$$
\frac{k_{o} d\left(n_{s}^{2}-n_{e}^{2}\right)^{3 / 2}}{3 n_{s} \Delta n}=m \pi+\pi / 4+\phi_{3}
$$

where

$$
\varphi_{3}=\tan ^{-1} \xi \sqrt{\left[\frac{n_{e}^{2}-n_{3}^{2}}{n_{s}^{2}-n_{e}^{2}}\right]}
$$

where $\zeta=1$ for TE and $\left(\mathrm{n}_{\mathrm{s}} / \mathrm{n}_{3}\right)^{2}$ for TM modes

These equations are solved theoretically from the experimental data by writing a simple computer programme to obtain the refractive index difference $\Delta \mathrm{n}$, the surface index $n_{s}$ and the effective depth of the waveguide, $d$.

The principal benefits of this experiment are additional insight into propagating modes in dielectric guides and some exposure to elementary fabrication procedures. Additionally the observation that the guide effective index varies with mode number immediately gives rise to the concept of intermode dispersion - a change in effective index implies a commensurate change in modal group and phase velocities. These concepts can then feed into the communications experiment outlined very briefly below.

\section{EXPERIMENTAL INTRODUCTION TO OPTICAL COMMUNICATION SYSTEMS}

The objective of this experiment is to provide a cost effective demonstration of the principal features of an optical fibre communication system. In particular, the experiments should require only normally available undergraduate teaching equipment such as power supplies, standard (20MHz) oscilloscopes and signal generators with comparable bandwidths.

The laboratory equipment comprises the following items :

- separate light emitting diode and laser transmitter modules with electrical inputs for modulation signals and fibre optic connector outputs

- $\quad$ a PIN diode detector module provided with an output to display on the oscilloscope

- a $\quad$ a $1.4 \mathrm{~km}$ length of step index, hard clad silica optical fibre selected for its high dispersion (both material and intermode) and high attenuation coefficients

- $\quad 1 \mathrm{~m}$ optical fibre jumper leads to connect the $1 \mathrm{~km}$ fibre reel to the transmit and receive modules and also to interconnect transmit and receive modules to obtain a direct interconnect reference signal. A diagram of the system is shown in Figure 4.

During the course of the experiment, students undertake the following :

- measurement of the current:fibre launched power characteristics of the LED and laser diode transmitter modules when connected directly to the receiver using the $1 \mathrm{~m}$ jumper cables 
- $\quad$ measurement of transmitter and receiver frequency and step function responses using both LED and laser sources again interconnected using the $1 \mathrm{~m}$ jumper lead

- interconnection of the $1 \mathrm{~km}$ link between transmitter and receivers and from this, measurement of the attenuation coefficient of the fibre (including the effects of connectors)

- $\quad$ measurement of the frequency response and step function response of the $1 \mathrm{~km}$ optical link using both the LED and laser sources

- measurement of the receiver noise level and sensitivity (referred to the displays within the PIN diode receiver which were previously calibrated against a power meter).

These measurements, of which the examples are shown in figures 5-7 are then used by the students to interpret the performance potential offered by the communications link. The objectives of these calculations are to highlight the various contributions to dispersion in optical fibres and to examine the impact of dispersion and attenuation on the limiting performance on an optical fibre link.

The frequency domain measurements (figure 5) for the laser diode transmission system illustrate a system electrical bandwidth of $12 \mathrm{MHz}$ which converts (see appendix) to an effective rise time of $40 \mathrm{nsec}$ or a dispersion of $36 \mathrm{nsec}$. These measurements are taken over a $1.4 \mathrm{~km}$ length of fibre so that the bandwidth:distance product is approximately $17 \mathrm{MHz} . \mathrm{km}$ and the dispersion is - assuming a linear relationship between length and dispersion $-26 \mathrm{nsec} / \mathrm{km}$.

In contrast the LED source results - presented in figure 7 as rise time observations - show a 10:90\% pulse rise time of $65 \mathrm{~ns}$ or a dispersion of $39 \mathrm{nsec} / \mathrm{km}$ translating to an equivalent bandwidth of $11.4 \mathrm{MHz} . \mathrm{km}$.

Examining the dispersion in the two cases and assigning the differential to material dispersion gives a value of about $29 \mathrm{nsec} / \mathrm{km}$ for the material dispersion within the bandwidth of the $830 \mathrm{~nm}$ LED source equivalent to a dispersion (for a $50 \mathrm{~nm}$ linewidth) of 560 picosec $/ \mathrm{km} . \mathrm{nm}$. This is somewhat higher than the normal value for pure silica but the dispersion has been exacerbated by the hard clad silica coating and the (unknown) dopants and impurities within the particular sample of fire which was chosen. Whilst it is within the range of manufacturers' values for fibres of this type, there appear to be contributions due to differential mode fill.

The measured attenuation for the fibre link is $14 \mathrm{~dB}$ - equivalent to $10 \mathrm{~dB} / \mathrm{km}$ (note that this is the attenuation for optical power which is proportional to the received voltage). The students are encouraged to estimate the noise level (acknowledging that this estimate is very rough) from the oscilloscope traces and typically this gives, after examining the trace with no optical input, a voltage signal to noise ratio of approximately 100 for the LED source. The laser source typically launches an additional $10 \mathrm{~dB}$ of optical power so that the equivalent, again voltage, signal to noise ratio at the receiver would be approximately 1000:1. 
We now have all the necessary information to project system performance for both laser and LED sources - but we do need to make some assumptions about the necessary received signal to noise ratio and also to remember the assumptions concerning the receiver bandwidth set at $20 \mathrm{MHz}$ by the use of laboratory oscilloscope.

Assuming that the noise level remains constant (in other words we do not alter the bandwidth) and that a $10 \mathrm{~dB}$ signal to noise ratio is adequate for a digital system ( $\mathrm{SNR}=12$ gives a b.e.r. of $\left.10^{-9}\right)$, then only an additional $10 \mathrm{~dB}$ of attenuation in the optical domain will be sufficient to reduce the receiver signal to noise ratio to this level for the LED system and $20 \mathrm{~dB}$ for the laser system. The total available transmission lengths together with their equivalent total dispersion are indicated in Table 1.

Table 1: System Performance Summary

\begin{tabular}{|c|c|c|c|c|}
\hline & Bandwidth & Attenuation & $\begin{array}{l}{ }^{*} \text { Max. } \\
\text { Transmission } \\
\text { Distance } \\
\end{array}$ & $\begin{array}{ll}\text { *Dispersion } & \\
\text { Limited } & \text { Total } \\
\text { Bandwidth } & \\
\end{array}$ \\
\hline $\begin{array}{ll}\text { Laser } & \text { Source } \\
780 \mathrm{~nm} & \\
\end{array}$ & $17 \mathrm{MHz} . \mathrm{km}$ & $10 \mathrm{~dB} / \mathrm{km}$ & $3.4 \mathrm{~km}$ & $5.8 \mathrm{MHz}$ \\
\hline LED Source $860 \mathrm{~nm}$ & $11.4 \mathrm{MHz} . \mathrm{km}$ & $10 \mathrm{~dB} / \mathrm{km}$ & $2.4 \mathrm{~km}$ & $4.75 \mathrm{MHz}$ \\
\hline & & & \begin{tabular}{lr}
$* 10 \mathrm{~dB}$ & \multicolumn{2}{c}{ receiver } \\
voltage & SNR in \\
$20 \mathrm{MHz}$ &
\end{tabular} & \\
\hline
\end{tabular}

Note: Clearly a narrower receiver bandwidth could increase transmission distance but would also impose a further bandwidth penalty

The comparison between these very modest results and those obtained in practical systems where $1 \mathrm{Gbit} / \mathrm{sec}$ over $100 \mathrm{~km}$ can be achieved illustrates graphically the range of optical fibre communications systems which may be available and students are invited to comment upon this distinction by considering both intermode dispersion effects (which are even present in single mode fibres though at a very low level) and the impact of source bandwidth and frequency chirping induced by switching the current in a narrow linewidth laser diode. The receiver in this experiment is also designed (deliberately) to have a somewhat poor noise performance and the impact of this upon system characteristics is significant. In the ideal world we would demonstrate these principles with high performance systems. Indeed whilst the unit cost of the fibre of such systems is typically far less than that used in our own system, the surrounding high frequency electronics, the need for very long lengths and a host of other factors preclude the application of such equipment for teaching purposes.

\section{CONCLUSIONS}

These two experiments illustrate within a viable laboratory cost and student time budget, the essential principles of optical communication systems. They are designed as independent but coupled mini projects and require typically in total 3-4 student days in the laboratory with the students working in groups of two or three. 
The principles of optical waveguiding in dielectric media, of polarisation dispersion and of resonant coupling are illustrated in the waveguide experiment and these concepts lead into the extremely important topic of dispersion in optical fibres when used in communication systems. In all optical systems the dispersion whether intermode or chromatic is due to multipath effects at the optical carrier frequency. In most electrical communication systems dispersion is due to differential time delays within the frequency spectrum of the signal which is being transmitted: the point to point link through electrical interconnection is almost always single path.

The communications experiment also allows -albeit rather crude - estimates of noise limited performance and dispersion limited performance within the system which is under investigation. There is also the provision for suggesting design improvements (typically receiver sensitivity, noise levels and receiver bandwidth) which may enhance the performance of these basic components yet further.

\section{APPENDIX - DISPERSION IN OPTICAL FIBRES}

The phenomenon of dispersion is most simply viewed in terms of the impulse response - indicated in figure 8. If we inject an extremely short pulse of light into the optical fibre it will later emerge as a broad pulse and the half power width of this pulse can be defined as a dispersion introduced by the fibre within the distance, L. For most fibre systems the value of $\tau_{d}$ the pulse dispersion increases approximately linearly with $\mathrm{L}$. In terms of measurable quantities the input may be determined by the modulation current applied to the source (assuming that it is operating in a linear regime - which it usually is) and the output from the photodiode detector current.

Normally the dispersion is observed either in terms of a frequency response introduced by the fibre or in terms of a deterioration in pulse rise time. Consequently, we need to transform the idealised response in figure 8 into these measurable quantities. The detailed arguments depend upon the exact pulse shape which emerges from the fibre and this in turn depends upon the detailed dispersion mechanisms and also on (for multimode fibre) the launch conditions. To simplify the condition we shall assume that the pulse shape is a Gaussion given by :

$$
P(\tau)=\frac{1}{\sigma \sqrt{2 \pi}} e^{-\tau^{2} / 2 \sigma^{2}}
$$

where $P$ is the optical power as a function of time and $\sigma$ is the standard deviation which is related to the dispersion time through :

$$
\tau_{d}=2.34 \sigma
$$

In order to obtain the step response of this system, the simplest approach is to convolve the impulse response with a unit step function (figure 9) which gives an error function rise time for which the distance between the 10 and $90 \%$ points is given by $\tau_{\mathrm{r}}$ :

$$
\tau_{r}=2.56 \sigma
$$


The pulse dispersion and this rise time are then related by :

$$
\tau_{d}=0.91 \tau_{r}
$$

The frequency response (i.e. the ratio of the photodetector current to the laser drive current under linear modulation conditions) can be obtained from the Fourier transform of the impulse response :

$$
R(f)=e^{-2 \pi^{2} \sigma^{2} f^{2}}
$$

Where $\mathrm{f}$ is the modulation frequency and $\mathrm{R}$ is the ratio of output to input optical powers neglecting attenuation effects. This ratio (of currents) is one half the zero frequency value when :

$$
f_{1_{2}}=\frac{0.187}{\sigma}=\frac{0.438}{\tau_{d}}=\frac{0.479}{\tau_{r}}
$$

Therefore, we can relate - for the Gaussion pulse - the pulse rise time, the frequency response half voltage point and the fibre dispersion. These relationships are used in the main text of this paper but it must be emphasised that they are applicable in detail only to the Gaussion pulse. Other pulse shapes will produce somewhat different results. The relationships between all the constants are derived from standard tables of the Gaussion and the error function.

In optical fibres both intermode and material dispersion take place and the effects of these two processes should be added. The processes may each be characterised by their own dispersion time, $\tau_{\mathrm{I}}$ and $\tau_{\mathrm{m}}$. Again assuming Gaussion phenomena the variances should be added :

$$
\tau_{d}=\left(\tau_{i}^{2}+\tau_{m}^{2}\right)^{12}
$$

Material dispersion (i.e. the differing velocities of different optical frequencies) is almost always dominated by the bandwidth of the optical source. Even at a $1 \mathrm{GHz}$ modulation bandwidth with 100 picosec/km.nm dispersion the effective material dispersion due to modulation is only approximately $0.6 \mathrm{picosec} / \mathrm{km}$. This is, of course, significant for very long distance, very high capacity systems.

Intermode dispersion may be estimated on the basis of the fibre geometry and for step index fibres. Taking the mean dispersion to be half that between the extreme rays simple geometry shows that :

$$
\tau_{m} \approx \frac{1250(N A)^{2}}{n^{2}} n \mathrm{sec} / \mathrm{km}
$$

This equation applies only to step index guides and again the detailed practical result depends critically upon launch conditions - the assumption is that all modes are equally excited and in the laboratory context this is not always straightforward to achieve. 


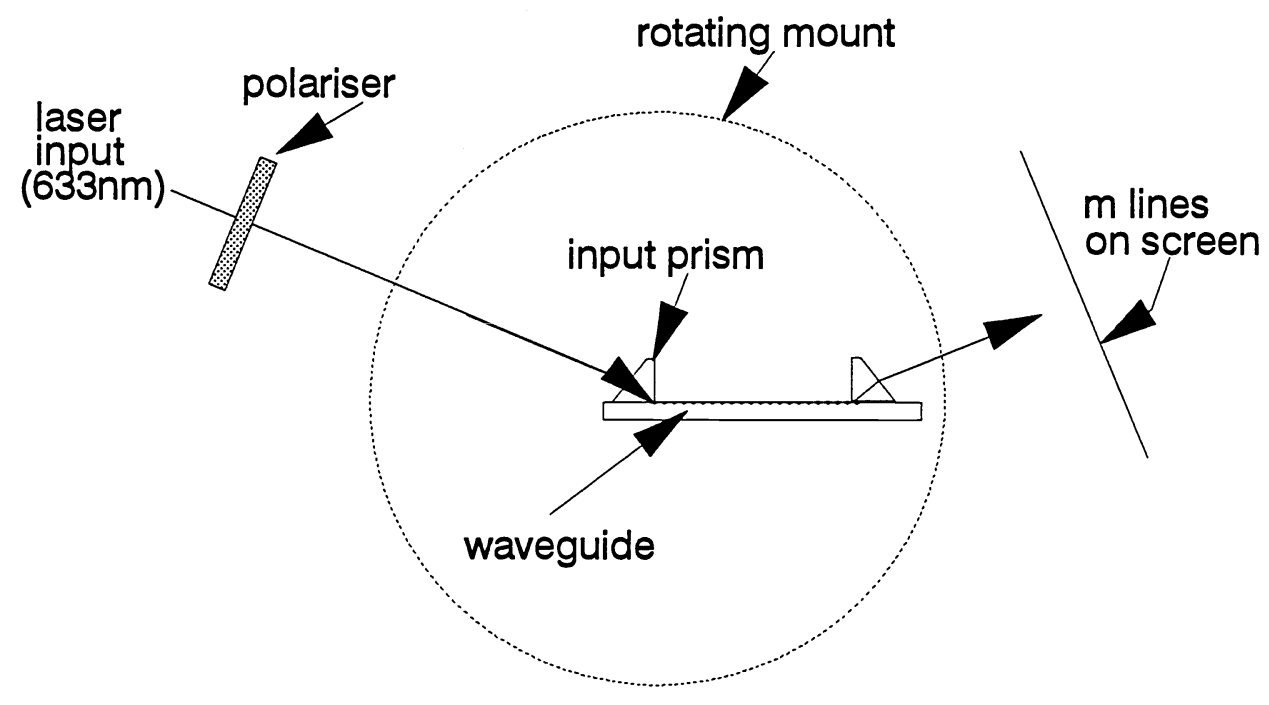

Figure 1 Optics for ion exchange waveguide experiment

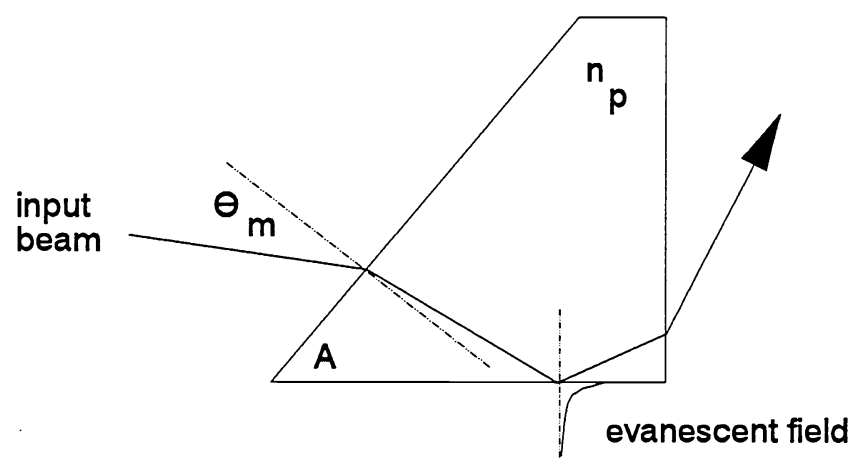

Figure 2 Launch conditions for prism coupling

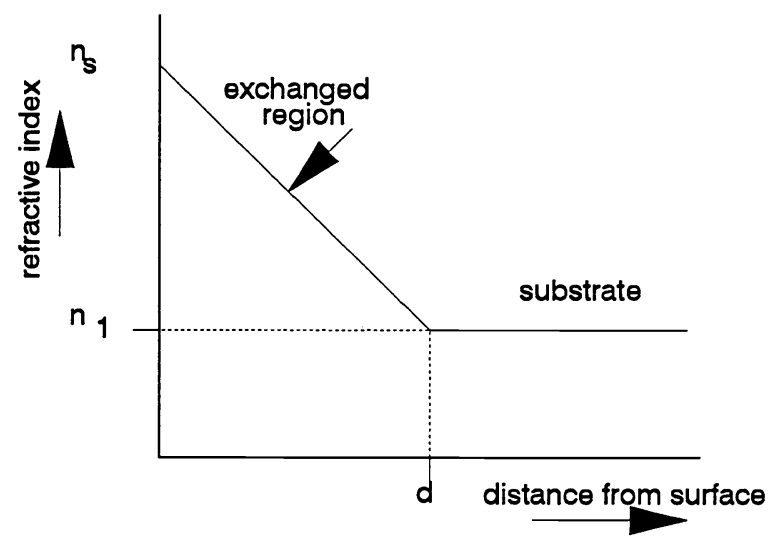

Figure 3 Refractive index profile approximation

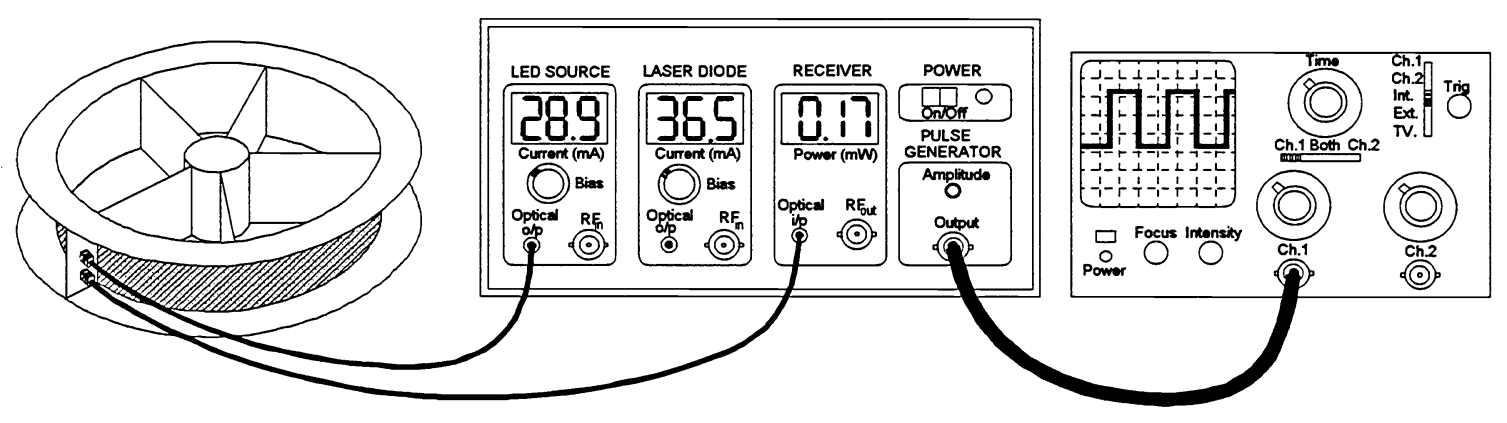

Figure 4 Schematic diagram of the communications demonstration experiment 

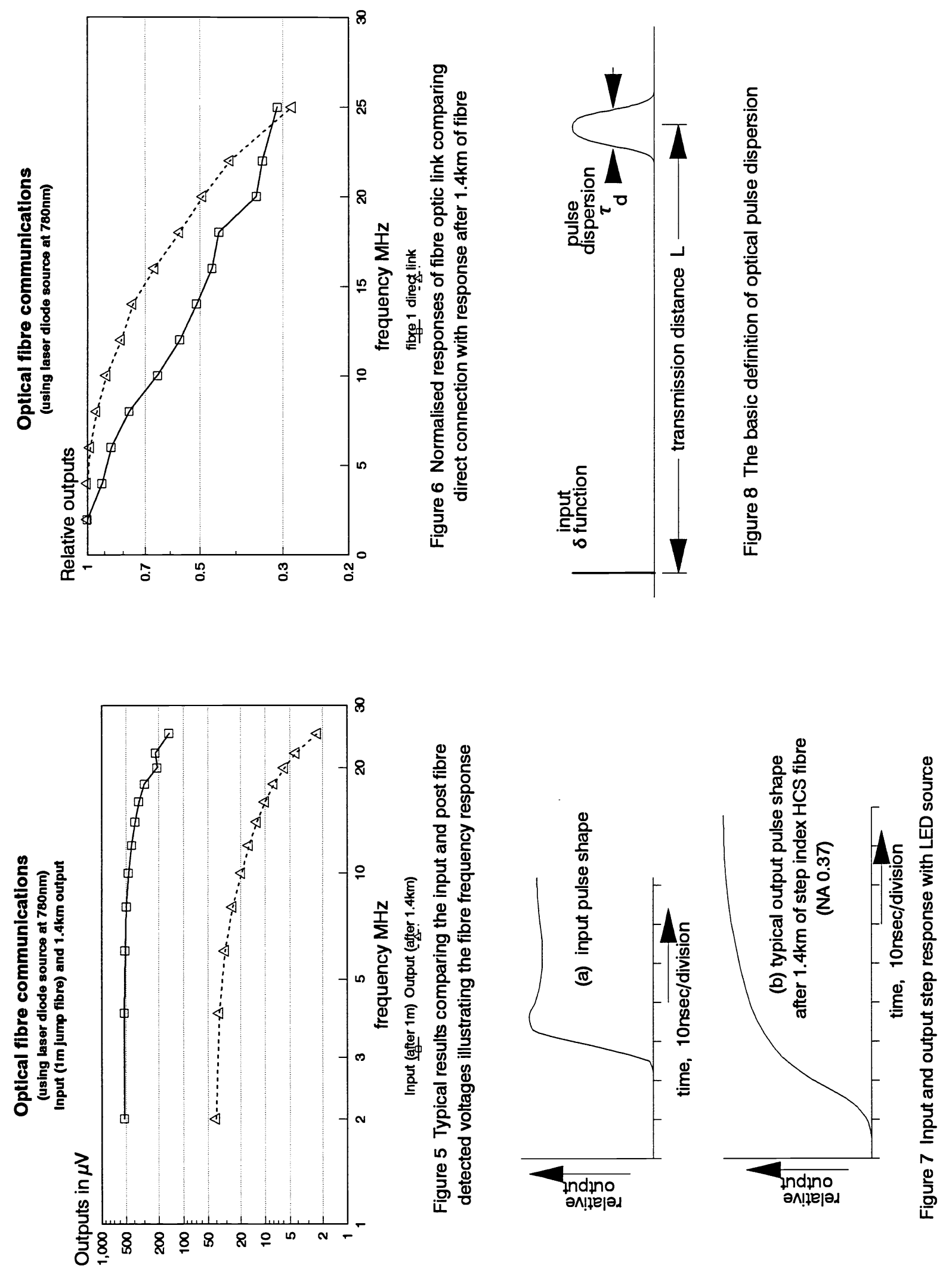

SPIE Vol. $2525 / 85$ 


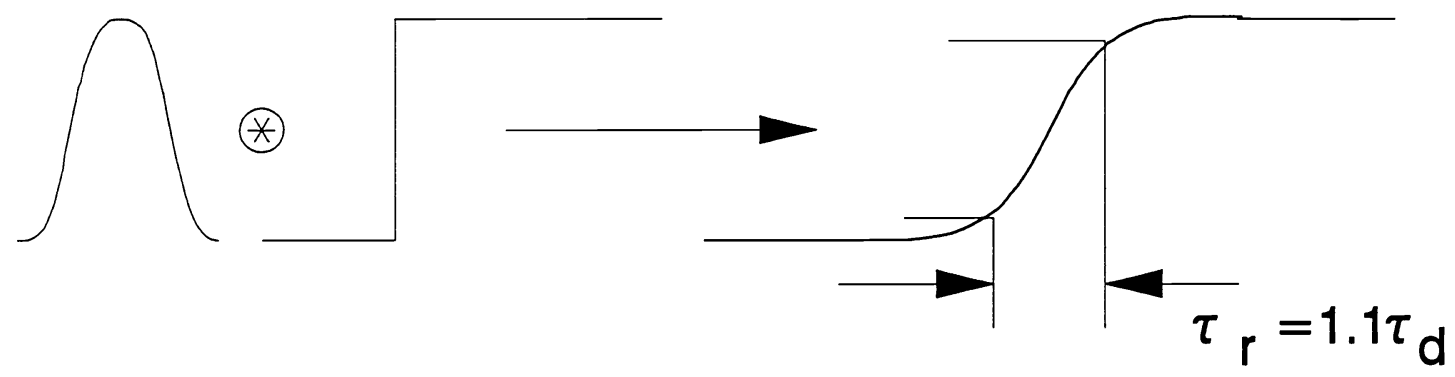

(a) convolving impulse response to yield the step response

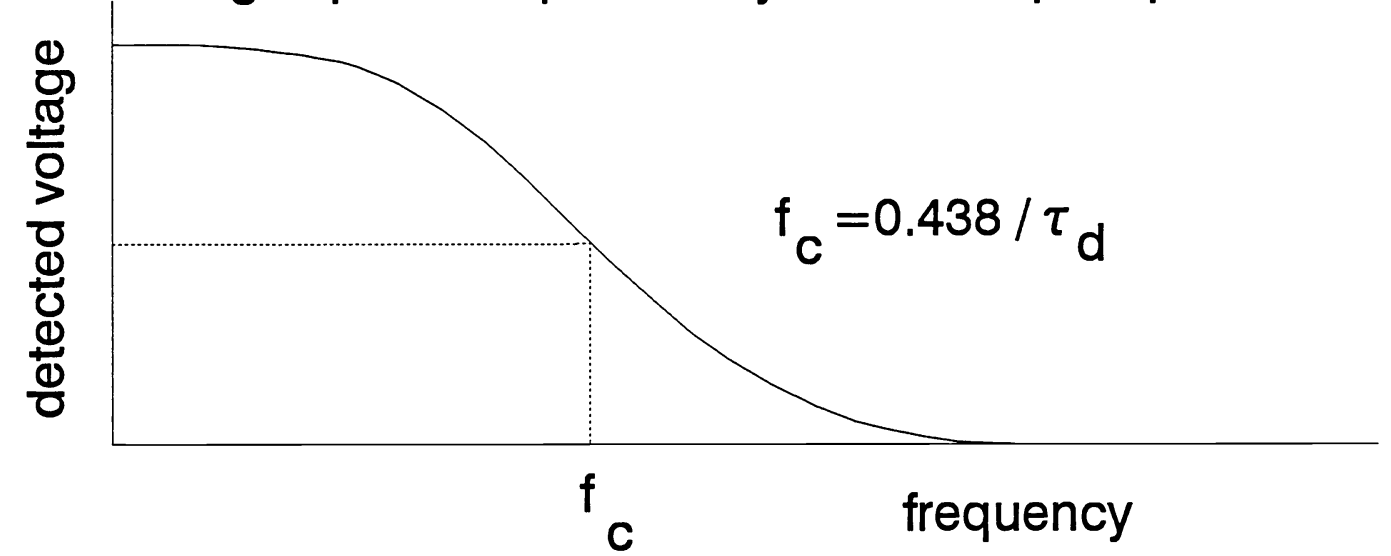

Figure 9 Relating step and frequency responses to dispersion time 